\section{MS15-P40 New luminescent materials based on ortho-phenylenediboronic acid - from crystal engineering to spectroscopic properties}

Katarzyna N. Jarzembska ${ }^{1}$, Radosław Kamiński ${ }^{1}$, Krzysztof Durka $^{2}$, Marcin Kubsik ${ }^{3}$, Krzysztof Nawara ${ }^{4}$

1. Czochralski Laboratory of Advanced Crystal Engineering, Biological and Chemical Research Centre, Department of Chemistry, University of Warsaw, Żwirki i Wigury 101, 02-089 Warsaw, Poland

2. Department of Chemistry, Warsaw University of Technology, Noakowskiego 3, 00-664 Warsaw, Poland

3. Department of Chemistry, University of Warsaw, Pasteura 1, 02-093 Warsaw, Poland

4. Institute of Physical Chemistry, Polish Academy of Sciences, Kasprzaka 44/52, 01-224 Warsaw, Poland

email: katarzyna.jarzembska@gmail.com

Arylboronic acids have already found many applications in diverse areas of chemical science, such as organic synthesis, catalysis or crystal engineering. Quite recently we have worked on para- and ortho-phenylenediboronic acids. ${ }^{1,2}$ In the present study, we focus our attention on a new class of adducts based on the latter compound.

It occurred that a simple one-pot reaction of ortho-phenylenediboronic acid with 8-hydroxyquinoline results in a new photoactive species. The reaction proceeds smoothly even in solvent-free conditions (mechanochemistry) and allows obtaining many modifications of such complexes (regarding both the acid and N-donor compound) in high yield. It was possible to crystallize and structurally characterize many of these systems, including several fluorinated derivatives and various solvatomorphs.

Additionally, luminescent properties of the studied complexes were investigated using the time-resolved spectroscopy, both in solution and in the solid state. The recorded emission spectra indicate the possibility of tuning the colour of the emitted light in the visible regime (from blue to orange). The determined lifetimes are of nanosecond order $(9-15 \mathrm{~ns})$, which suggests that the main contribution to the emitted light comes from the fluorescence process (singlet-to-singlet transition) what is further supported by the DFT calculations. Theoretical results show also that the charge transfer processes occur mostly in the quinoline ring fragment. The emission quantum yields determined in solution are in the range of 10 to $30 \%$. The lifetimes and quantum yield values are highly dependent on the number of fluorine substituents in the acidic part of the molecule. The more the F-substituents present, the lower the corresponding values.

[1] K. Durka, K. N. Jarzembska, R. Kamiński, S. Luliński, J. Serwatowski, K. Woźniak Cryst. Growth Des. 2012, 12, 3720 .

[2] K. Durka, K. N. Jarzembska, R. Kamiński, S. Luliński, J. Serwatowski, K. Woźniak, Cryst. Growth Des. 2013, 13, 4181.

Keywords: phenylenediboronic acids, quinoline derivatives, crystal engineering, fluorescence
MS16. Physical properties

through lattice distortions:

structure of perovskites \& Co

studied by electron microscopy

and diffraction

Chairs: Jerome Rouquette, Artem Abakumov

Nikolai N. Eremin ${ }^{1}$, Nikolai Protasov ${ }^{1}$, Oleksii Hrechanivskyi ${ }^{2}$

1. Moscow State University, Geological Faculty

2. Institute of Geochemistry, Mineralogy, and Ore Formation, National Academy of Sciences of Ukraine

\title{
email: neremin@mail.ru
}

The aim of this work was to investigate the relation between the radiation stability and the composition of $\mathrm{Ca}(\mathrm{Zr}, \mathrm{Ti}, \mathrm{Sn}) \mathrm{O}_{3}$ solid solution using method of molecular dynamics (MD). This compound with perovskite structure (space group Pbnm) is one of the promising matrices for utilization of high level radioactive waste. Note that a new mineral lakargiite, which represents the $\mathrm{Ca}(\mathrm{Zr}, \mathrm{Ti}, \mathrm{Sn}) \mathrm{O} 3$ solid solution with the maximal molar fraction $x\left(\mathrm{CaZrO}_{3}\right)=0.93$ and maximal fractions $x\left(\mathrm{CaTiO}_{3}\right)=0.22$ and $x\left(\mathrm{CaSnO}_{3}\right)=0.20$, was registered in 2007. Our original approach, described in [1] makes it possible to simulate the solid solutions using representative supercell of limited size with optimal atomic distribution. Thus, for MD simulation we used a supercell with different compositions with sizes $310 \times 210 \times 315 \AA$, which contains 1.4 million atoms with a maximally disordered distribution of $\mathrm{Zr}, \mathrm{Ti}$, and $\mathrm{Sn}$. As an analog of the recoil atom the knocked out Th atom with energy of $20 \mathrm{keV}$ was used. After the formation of the atomic displacement cascade at instant $t=0.71 \mathrm{ps}$ the structure starts to be "restored" and displaced atoms partially return to their sites or equivalent crystallographic sites. At the end of the simulation $(t=20$ $\mathrm{ps}$ ), the total number of Frenkel pairs is minimal (figure $1)$. In contrast to some other tested materials defects in $\mathrm{Ca}(\mathrm{Zr}, \mathrm{Ti}, \mathrm{Sn}) \mathrm{O} 3$ are mainly of the point character, which indicates the high stability of this solid solution to the radioactive effect. We evaluated parameter a, which characterized susceptibility to amorphization of material under radiation damage. This parameter numerically equal to a part of the energy of Th atom, which is consumed for the formation of Frenkel pairs in a cascade of displaced atoms. It was shown that the dependence of a-parameter on the solid solution composition is lowest for $\mathrm{Sn}-\mathrm{Zr}$ compositions, whereas Ti-riched compositions exhibit poorer performance. Thus the radiation stability of $\mathrm{Sn}-\mathrm{Zr}$-riches matrices can be of unquestionable interest 